\title{
The role of ofatumumab in the treatment of chronic lymphocytic leukemia resistant to previous therapies
}

This article was published in the following Dove Press journal:

Journal of Blood Medicine

24 February 2010

Number of times this article has been viewed

\section{Jorge Castillo}

Kimberly Perez

The Warren Alpert Medical School of Brown University, Division of Hematology and Oncology, The Miriam Hospital, Providence, RI, USA

\begin{abstract}
Chronic lymphocytic leukemia (CLL) is an indolent but incurable disease. Despite the improvement of the available therapies, the management of heavily-treated CLL patients represents a challenge for modern practitioners. Ofatumumab is a second-generation, fully human anti-CD20 monoclonal antibody that has shown activity in CLL patients who have failed very effective therapies such as fludarabine, alemtuzumab and rituximab. Potential benefits of ofatumumab include powerful complement-dependent cytotoxicity, less immunogenicity, faster infusions and activity in resistant CLL patients. Recently, the FDA has approved ofatumumab for the treatment of CLL patients who have failed fludarabine and alemtuzumab-based regimens. The aim of this review is to summarize the current knowledge regarding pharmacology, mechanism of action, pre-clinical and clinical development, and the role of ofatumumab for the treatment of CLL patients who have failed previous therapies. Further research is necessary to further define the role of ofatumumab in the treatment of CLL.
\end{abstract}

Keywords: ofatumumab, CLL, chronic lymphocytic leukemia, monoclonal antibodies, CD20

\section{Introduction}

\section{Management issues in the treatment of CLL}

Chronic lymphocytic leukemia (CLL) is a malignant lymphoproliferative disease that originates from a clonal proliferation of malignant B-lymphocytes. CLL is considered the most common adult leukemia in the Western Hemisphere and it tends to affect males more frequently than females. In 2009, it was estimated that there were 15,490 new cases diagnosed with 4390 associated deaths in the United States. ${ }^{1}$ The median age of presentation is 70 years, with a steady increase in risk of developing CLL with increasing age.

The clinical course of CLL is highly variable. Many patients are diagnosed at early, asymptomatic phases of the disease and are managed by expectant surveillance. ${ }^{2}$ A substantial portion of patients, however, will eventually need therapy and the course of their disease will be characterized by episodes of remissions and relapses that can last for several years. A smaller subset of patients will have high-risk or refractory disease with survival of only a few years. The clinical presentation of CLL has been linked with prognostic significance; early stage disease has an estimated life expectancy of greater than 10 years and advanced stage disease has an estimated life expectancy of 18 months to 3 years. The final prognosis of CLL patients has been linked to the presence of several features, including immunoglobulin variable region heavy-chain $\left(\mathrm{IgV}_{\mathrm{H}}\right)$ mutational status, expression of CD38 and/or ZAP-70, and cytogenetic abnormalities. ${ }^{3}$
The Miriam Hospital, I64 Summit Ave,

Fain Building, Providence, RI 02906, USA

Tel + I 40I-7937|5I

Fax $+\mid$ 40I-7937|32

Email jcastillo@lifespan.org 
The diagnosis of CLL is established by the presence of B-cell lymphocytosis of greater than 5000 cells $/ \mu \mathrm{L}$ in the peripheral blood for more than 3 months. ${ }^{2}$ The leukemic cells are small, mature-appearing lymphocytes with scant cytoplasm and dense nuclei lacking discernible nucleoli. The characteristic immunophenotype of CLL shows a coexpression of CD5, a T-cell antigen, and the B-cell antigens CD19, CD20 and CD23. The levels of expression of CD20 and CD79b are characteristically low when compared to normal mature B-cell populations. ${ }^{4}$ The monoclonality of the population can be determined by surface immunoglobulin light chain restriction, detection of clonal cytogenetic abnormalities and/or clonal immunoglobulin gene rearrangements. In $80 \%$ of the cases, molecular genetic lesions can be detected at time of diagnosis. Common genetic abnormalities found in CLL include 13q deletion, trisomy 12 and $11 \mathrm{q}$ and $17 \mathrm{p}$ deletions; deletions in $11 \mathrm{q}$ and $17 \mathrm{p}$ are associated with poorer prognosis. ${ }^{5}$

Currently there are 4 drugs approved by the Food and Drug Administration (FDA) for use in patients with CLL: chlorambucil (Leukeran $^{\circledR}$; GlaxoSmithKline, Research Triangle Park, NC, USA), fludarabine (Fludara ${ }^{\circledR}$; Ben Venue Laboratories, Bedford, OH, USA), bendamustine (Treanda ${ }^{\circledR}$; Cephalon Inc., Frazer, PA, USA) and alemtuzumab (Campath ${ }^{\circledR}$; Genzyme Corporation, Cambridge, MA, USA). Chlorambucil, an alkylating agent, was the first drug approved for CLL and is effective on inducing responses lasting for 1 to 2 years. Fludarabine has achieved higher response rates than chlorambucil in randomized controlled trials; however, the benefit on progression-free survival (PFS) in the elderly is less clear. ${ }^{6}$ Fludarabine is also associated with higher rates of severe infections and neutropenia. ${ }^{7}$ Bendamustine has shown to be superior to chlorambucil in obtaining clinical responses and prolonging PFS with an acceptable toxicity profile. ${ }^{8}$ Alemtuzumab is an anti-CD52 monoclonal antibody $(\mathrm{MAb})$ approved as frontline and second-line therapy for CLL. Alemtuzumab has shown efficacy in high-risk patients carrying $17 \mathrm{p}$ deletions $\mathrm{s}^{9,10}$ and to eradicate minimal residual disease. ${ }^{11}$ Unfortunately, alemtuzumab is associated with profound myelo and immunosuppression resulting in an increased rate of infections such as CMV reactivation. ${ }^{12}$

Rituximab (Rituxan ${ }^{\circledR}$; Genentech, South San Francisco, CA, USA) is a chimeric antiCD20 MAb and, although is not FDA-approved, it is the MAb most commonly used in CLL and has shown improvements in overall response rates (ORR) and progression-free survival (PFS) when added to chemotherapy in large randomized trials. ${ }^{13,14}$ The response to rituximab, however, seems to decrease with subsequent rituximab-containing regimens and, although rarely, the administration of rituximab has been associated with life-threatening adverse events, such as anaphylactic and severe mucocutaneous reactions.

Newer agents that have shown efficacy in CLL are flavopiridol and lenalidomide. Flavopiridol is a cyclin-dependent kinase inhibitor that showed efficacy on inducing apoptosis in CLL lines. ${ }^{15}$ In a recent phase II trial, flavopiridol induced responses in $53 \%$ of the treated patients with a median overall survival (OS) of 8.6 months. ${ }^{16}$ The most common grade 3-4 adverse events were neutropenia (81\%), diarrhea (72\%), tumor lysis syndrome (22\%) and liver enzymes levels elevation (34\%). Lenalidomide is an immunomodulator agent with a wide antitumor activity, which has shown to alter cytokine production, modulate effector cell responses and directly induce apoptosis in malignant cells. ${ }^{17,18} \mathrm{~A}$ phase II trial in relapsed or refractory CLL showed an ORR of $47 \%$ with a median OS that was not reached at the time of publication. ${ }^{19}$ The most common grade 3 or 4 adverse events were neutropenia (70\%), thrombocytopenia (45\%), flare reaction (8\%), pulmonary embolism (5\%) and tumor lysis syndrome (5\%).

Despite newer drug options, the disease remains incurable with current standard therapies. Over the last decade, prospective, randomized trials have analyzed various combinations of nucleoside analogues, alkylating agents and MAbs for the treatment of CLL. It has been demonstrated that as additional agents were added to a fludarabine-based regimen, response rates, with ORR and complete response (CR) rates in excess of $90 \%$ and $50 \%$, respectively, and PFS have improved; however, improved response rates have not translated into longer OS (see Tables 1 and 2 for selected CLL regimens used in the front-line and relapsed settings, respectively).

Areas of investigation to improve the current therapy include patient populations with high-risk disease, based on IgVH mutational status, ZAP-70 and/or CD38 expression and molecular cytogenetics, and those with relapsed or refractory disease. Patients that have become resistant to fludarabine and alemtuzumab-based regimens, also called double refractory (DR), have an associated median survival of less than 1 year, low response rates to salvage therapy and increased rate of adverse events. ${ }^{20}$ In a similar manner, bulky fludarabine-resistant disease (BFR) has also been associated with poor responses, reduced survival and increased rate of toxicity, mainly of an infectious nature. ${ }^{20}$

\section{Role of ofatumumab}

To address the need for improved response and overall survival rates, many other agents have been evaluated. 
Table I Selected regimens used as first-line therapy in chronic lymphocytic leukemia

\begin{tabular}{|c|c|c|c|c|c|}
\hline Author & Regimen & No patients & ORR/CR & Progression-free survival & Overall survival \\
\hline \multirow[t]{2}{*}{$\mathrm{Rai}^{7}$} & Fludarabine $(\mathrm{F})$ & 170 & $63 \% / 20 \%$ & Median 20 months & Median 66 months (NS) \\
\hline & Chlorambucil & 181 & $37 \% / 4 \%$ & Median 14 months & Median 55 months \\
\hline \multirow[t]{2}{*}{ Hillmen ${ }^{36}$} & Alemtuzumab (A) & 149 & $83 \% / 24 \%$ & Median I 4.6 months & 2-year 84\% (NS) \\
\hline & Chlorambucil & 148 & $55 \% / 2 \%$ & Median II.7 months & 2 -year $84 \%$ \\
\hline \multirow[t]{2}{*}{ Knauf ${ }^{8}$} & Bendamustine (B) & 162 & $68 \% / 31 \%$ & Median 21.6 months & NS \\
\hline & Chlorambucil & 157 & $31 \% / 2 \%$ & Median 8.3 months & NS \\
\hline \multirow[t]{2}{*}{ Hallek $^{13,37}$} & FCR & 408 & $95 \% / 44 \%$ & Median 52 months & 3 -year $84 \%$ \\
\hline & FC & 409 & $88 \% / 22 \%$ & Median 33 months & 3 -year $79 \%$ \\
\hline \multirow[t]{3}{*}{ Robak $^{38}$} & CM + cladribine & 163 & $80 \% / 36 \%$ & Median 23 months & Median not reached (NS) \\
\hline & C + cladribine & 171 & $83 \% / 29 \%$ & Median 22 months & Median not reached \\
\hline & Cladribine & 174 & $77 \% / 21 \%$ & Median 23 months & Median 51 months \\
\hline $\mathrm{Kay}^{39}$ & PCR & 65 & $91 \% / 41 \%$ & Median 32.6 months & NR \\
\hline Parikh $^{40}$ & CFAR & 60 & $92 \% / 70 \%$ & 2 -year $68 \%$ & NR \\
\hline Fischer ${ }^{41}$ & $\mathrm{BR}$ & 117 & $91 \% / 33 \%$ & Median not reached & NR \\
\hline
\end{tabular}

Abbreviations: C, cyclophosphamide; R, rituximab; M, mitoxantrone; ORR, overall response rate; CR, complete response; NS, not statistically significant; NR, not reported.

Ofatumumab (Arzerra ${ }^{\mathbb{}}$; GlaxoSmithKline, Collegeville, PA, USA and Genmab, Copenhagen, Denmark) is a second-generation, fully human antiCD20 MAb whose target epitope is distinct from rituximab. Several clinical trials in patients with relapsed DR or BFR CLL have demonstrated promising response rates with a safe toxicity profile and have established ofatumumab as a possible therapeutic option. The aim of this paper is to summarize the current knowledge and discuss potential applications of ofatumumab in patients with relapsed CLL who have failed previous therapies.

\section{Review of pharmacology, mode of action, pharmacokinetics of ofatumumab}

Ofatumumab is an IgG1, fully human, second-generation antiCD20 MAb with a molecular weight of $150 \mathrm{kDA}$. It was produced by immunizing $\mathrm{HCo} 7$ and $\mathrm{KM}$ mice with a murine cell line transfected with human heavy and light chain genes. MAbs exert their therapeutic effect by enhancing antigendependent cellular cytotoxicity (ADCC) and complementdependent cytotoxicity (CDC) while inducing apoptotic

Table 2 Selected regimens used in relapsed or refractory chronic lymphocytic leukemia

\begin{tabular}{|c|c|c|c|c|c|}
\hline Author & Regimen & \# Patients & ORR/CR & $\begin{array}{l}\text { Progression-Free } \\
\text { Survival }\end{array}$ & Overall Survival \\
\hline \multirow[t]{2}{*}{ Robak $^{14}$} & FCR & 276 & $70 \% / 24 \%$ & Median 31 months & Median not reached (NS) \\
\hline & $\mathrm{FC}$ & 276 & $58 \% / 13 \%$ & Median 21 months & Median 53 months \\
\hline \multirow[t]{2}{*}{ O’Brien ${ }^{42,43}$} & FC + oblimersen & 120 & NR/9\% & NR & 5 -year 25\% (NS) \\
\hline & FC & 121 & $N R / 3 \%$ & NR & 5 -year 15\% \\
\hline Fischer ${ }^{44}$ & $B R$ & 81 & $77 \% / 15 \%$ & NR & NR \\
\hline Byrd 45 & FCR + lumiliximab & 31 & $65 \% / 52 \%$ & Median 19 months & NR \\
\hline Lamanna $^{46}$ & PCR & 32 & $75 \% / 25 \%$ & NR & Median 44 months \\
\hline $\operatorname{Lin}^{16}$ & Flavopiridol & 64 & $53 \% / 2 \%$ & Median 9 months & NR \\
\hline Chanan-Khan ${ }^{19}$ & Lenalidomide & 45 & $47 \% / 9 \%$ & Median not reached & NR \\
\hline Coiffier $^{27}$ & Ofatumumab & 33 & $42 \% / N R$ & NR & NR \\
\hline Kipps $^{30}$ & Ofatumumab & 138 & $47 \%-58 \% / N R$ & NR & Median I4-I5 months \\
\hline Wierda $^{32}$ & $\mathrm{FC}+$ ofatumumab & 61 & $73 \%-77 \% / 32 \%-50 \%$ & Median not reached & NR \\
\hline
\end{tabular}

Abbreviations: F, fludarabine; C, cyclophosphamide; R, rituximab; B, bendamustine; P, pentostatin; ORR, overall response rate; CR, complete response; NS, not statistically significant; NR, not reported. 
pathways within the target cells. ${ }^{21}$ Ofatumumab binds to a different epitope on the CD20 molecule than rituximab, which is located on the smaller extracellular loop of CD20 (Figure 1) and then releases very slowly from the target (reduced off-rates). ${ }^{22}$ These two characteristics are thought to be the etiology of its higher efficacy when compared to rituximab in pre-clinical models.

Teeling and colleagues demonstrated that ofatumumab was able to stimulate cell killing when incubated with plasma alone, therefore confirming its role as a strong CDC inducer. Furthermore, cell killing was blunted after heat-induced complement inactivation. In this same study, the group demonstrated that rituximab mixed with CLL cells with low CD20 expression (Raji) showed no activity, while ofatumumab when exposed to Raji cells demonstrated activity. ${ }^{23}$

A follow-up study compared the required CD20 concentrations for activity between rituximab and ofatumumab. ${ }^{24}$ The results of their analysis established that ofatumumab achieved full lysis with CD20 concentrations as low as 4,500 molecules compared with rituximab, which required at least 30,000 molecules to be active. As part of this study, on- and off-rates of rituximab and ofatumumab were compared. The on-rates were similar with both MAbs but the off-rates were longer with ofatumumab. Finally, when blocking the A170/P172 epitope, the rituximab binding site located in the extracellular domain of the large loop of the CD20 molecule, the effect of ofatumumab was not affected. From these studies, the authors concluded that the ability of ofatumumab to elicit a stronger CDC should be associated to the recognition of a different form of CD20 or the possible binding to a different epitope within the CD20 molecule. Using epitope mapping, ofatumumab showed binding to an epitope located in the extracellular domain of the small loop of CD20.

Beum and colleagues, using a microscopic analysis in which different lymphoma cell lines undergo opsonization with rituximab and ofatumumab, showed that morphologic membranous changes such as 'blebbing' and 'streamers', which are directly associated with complement-induced death, appeared more quickly with ofatumumab-exposed cell lines than with rituximab (114 vs 418 seconds, respectively). Importantly, ofatumumab showed activity against cell lines with high levels of CD55 and CD59, which are complement

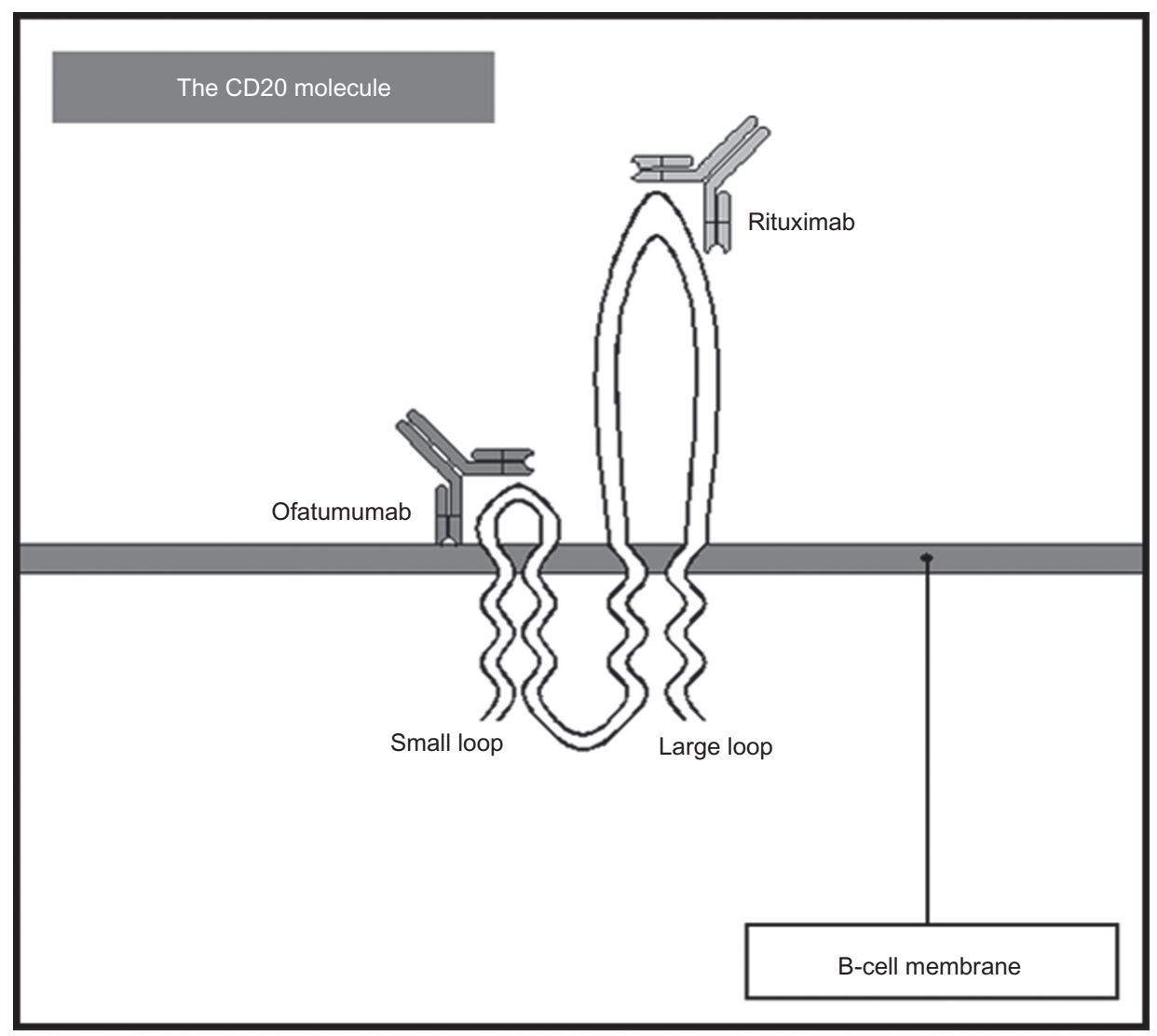

Figure I The CD20 molecule structure. Ofatumumab binds to a different epitope (small loop) than rituximab (large loop). 
inactivating molecules; rituximab did not show activity even after several hours of incubation. ${ }^{25}$

Pharmacokinetic studies with ofatumumab were undertaken in lymphoma cell lines, mice and monkeys. ${ }^{26}$ Using Daudi cells, CDC was seen at levels of $0.13 \mu \mathrm{g} / \mathrm{mL}$ with a full target saturation achieved at levels of $5 \mu \mathrm{g} / \mathrm{mL}$; no further increase in CDC was seen with higher doses of ofatumumab. In a xenograft model, control mice developed lymphoma 13 days after injection of Daudi cells while lymphoma developed 3 to 4 weeks later in mice exposed to ofatumumab at a dose of $0.5 \mathrm{mg} / \mathrm{kg}$. Tumor growth was seen when ofatumumab levels were less than $0.4 \mu \mathrm{g} / \mathrm{mL}$. In Cynomolgus monkeys, which have a CD20 molecule very similar to humans, a total B-cell depletion was observed immediately after exposure to ofatumumab with a full recovery of B-cells in 96 to 136 days. The authors concluded that concentrations of $50 \mu \mathrm{g} / \mathrm{dL}$ and 5 to $10 \mu \mathrm{g} / \mathrm{dL}$ were needed to obtain and maintain the effect of ofatumumab in B-cells, respectively.

\section{Safety and tolerability of ofatumumab}

A phase I/II trial conducted by Coiffier and colleagues addressed the issue of safety and tolerability of ofatumumab in relapsed or refractory CLL. ${ }^{27}$ This trial analyzed 33 patients and divided them into 3 cohorts receiving different doses of ofatumumab; cohort $A(n=3)$ received the first infusion of $100 \mathrm{mg}$ and 3 subsequent infusions of $500 \mathrm{mg}$; cohort $B$ $(\mathrm{n}=3)$ received a first infusion of $300 \mathrm{mg}$ and 3 subsequent infusions of $1000 \mathrm{mg}$; and cohort $\mathrm{C}(\mathrm{n}=27)$ received a first infusion of $500 \mathrm{mg}$ and 3 subsequent infusions of $2000 \mathrm{mg}$. The large majority of the patients (97\%) received all four infusions. The results of the analysis demonstrated that 27 patients reported 246 adverse events of which 7\% were grade 3 or 4 . Of all the adverse events, $61 \%$ were deemed related to treatment, the majority of which were reported on the day of infusion. This toxicity was noted to be similar to rituximab-related infusion reactions in CLL patients. Other notable serious adverse events included infections (51\%) consisting of herpes zoster, pneumonia, sinusitis; hematologic toxicity (15\%) consisting of neutropenia and hemolytic anemia; angina pectoris $(3 \%)$; hepatitis $(3 \%)$; carotid artery stenosis (3\%); and interstitial lung disease $(3 \%)$. Of the serious toxicities (grade 3 and 4 ) only 5 were considered treatment-related, herpes zoster, neutropenia, interstitial lung disease and hepatitis.

In a phase II collaborative international effort, ${ }^{28}$ similar adverse effects were noted. The most common adverse events were first infusion-related reactions with an incidence of $38 \%$ to $46 \%$; the incidence of grade 3 events was $3 \%$ to $7 \%$. The incidence of infusion-related reactions decreases greatly with subsequent infusions. The most common grade 3 or 4 events were infections ( $25 \%$ to $27 \%$ ), neutropenia ( $10 \%$ to $12 \%$ ) and anemia (4\% to $8 \%$ ). Early death occurred in 5 patients but none were related to the administration of therapy. Although a potential concern, there was no evidence of development of human anti-human antibodies against ofatumumab.

\section{Efficacy studies}

\section{Phase I/II studies}

The initial dose-escalation study, Hx-CD20-402, was a phase I/II trial in which 3 doses of ofatumumab were administered to 33 patients with relapsed or refractory CLL. ${ }^{27}$ The results at the conclusion of this study demonstrated an overall response rate (ORR) at 19 weeks after therapy of $44 \%$. The ORR per cohort was as follows: Cohort A 33\% $(n=1)$, cohort B $0 \%$ and cohort C 50\% $(n=13)$. From the patients previously treated with rituximab $(n=7)$, alemtuzumab $(n=6)$ and or fludarabine $(n=20)$, seven responded to treatment. However, not all responses were sustained and at week 19 only 9 patients $(27 \%)$ had sustained responses. When response was analyzed by hematologic parameters: $6 / 7$ anemic patients showed improvement ( 1 cohort B and 5 cohort C); 8/9 had improvement with thrombocytopenia; bone marrow evaluation: 11 in cohort $\mathrm{C}$, of which 8 who demonstrated response to treatment the median percentage of marrow lymphocytes decreased from $78 \%$ to $50 \%$ after treatment and 3 patients had less than $30 \%$; and on analysis of the malignant population of CD5+/CD19+ B-cells in the periphery in cohort $\mathrm{C}$, there was a median $55 \%$ reduction observed after the first infusion; after the fourth infusion the percentage reduction from baseline was $97 \%$ (range $15 \%$ to $100 \%$ ) and sustained until week 24.

A second study, Hx-CD20-406, is an international multicenter phase II study that is currently ongoing but data from the planned 12-month interim analysis have been released. ${ }^{28-30}$ Ofatumumab was used in 59 patients with refractory DR and 79 patients with BFR CLL. In this study, patients received 8 weekly infusions of ofatumumab followed by 4 monthly infusions (Dose 1, $300 \mathrm{mg}$; Doses 2-12, $2000 \mathrm{mg}$ ); 54\% of the patients received all 12 infusions and $90 \%$ received 8 infusions. The median prior therapy exposure was 5 (DR) and 4 regimens (BFR), with a prior rituximab exposure of $59 \%$ (DR) and 43\% (BFR). The final results published included an ORR of $58 \%(n=34)$ for the DR group and $47 \%(n=30)$ for the BFR group. The median time until next CLL treatment was 8 to 9 months and the median OS was about 15.4 months 
for the DR group and 13.7 months for the BFR group. At the landmark analysis at week 12 , a clinical response significantly correlated with longer survival for both DR and BFR groups. Additionally, in patients not achieving a formal response by National Cancer Institute-sponsored Working Group (NCIWG) criteria, clinical benefit was observed with resolution of symptoms and improvement in thrombocytopenia and anemia. Interestingly, similar response rates were observed in CLL patients with or without prior exposure to rituximab.

Ongoing phase II trials include a re-treatment and maintenance with ofatumumab for patients who have progressed after an initial response to ofatumumab (NCT00802737) and the combination of ofatumumab with fludarabine and cyclophosphamide in previously untreated CLL patients (NCT00410163); ${ }^{31}$ the latter is a randomized phase II trial comparing 2 doses of ofatumumab, $500 \mathrm{mg}$ and $1000 \mathrm{mg}$, given in combination with fludarabine and cyclophosphamide. These combinations have shown great activity with ORR of $77 \%$ and $73 \%$ and CR rates of $32 \%$ and $50 \%$ for the 500-mg and 1000-mg arms, respectively. ${ }^{32}$ Planned phase II studies include the combination of ofatumumab and bendamustine (NCT01010568) and the combination of ofatumumab and lenalidomide (NCT01002755) in previously treated CLL patients. $^{31}$

\section{Phase III studies}

Two randomized controlled trials are ongoing. ${ }^{31}$ Chlorambucil with or without ofatumumab is being evaluated in untreated patients with CLL (COMPLEMENT 1; NCT00748189). Enrollment started in December 2008 with an estimated goal study population of 444 patients. The second study will evaluate the combination of fludarabine and cyclophosphamide with or without ofatumumab in previously treated CLL patients (NCT00824265). Enrollment started in March 2009 and aims to prospectively enroll 352 patients.

\section{Patient perspectives}

On October 26, 2009 the FDA announced the approval of ofatumumab for its use in patients with refractory or relapsed CLL that have failed fludarabine and alemtuzumab-based regimens. ${ }^{33}$ Since ofatumumab has a benign side effect profile along with lower rates of infusion-related reactions and faster infusions than rituximab, good compliance and higher patient satisfaction rates are expected.

\section{Conclusions}

As CLL remains an incurable disease with the current treatment options, the pursuit of newer drugs and combination regimens is warranted. The goal of our therapy in CLL should be focused on obtaining durable responses and prolonging survival while improving or, at least, not affecting the quality of life of the patients. Clinicians have at hand a myriad of different available therapies and the rational use of these could be a challenge and needs careful consideration and analysis. The available therapies for CLL will give evidencebased hematologists and oncologists the opportunity to put into practice what we call the art of medicine.

With an improved understanding of the biology of cancer, multiple pathways are being discovered, evaluated and targeted with drugs struggling to be tried in well-planned studies. Unfortunately, only a handful of these regimens will be valued enough to get into large randomized trials. The CD20 molecule has arisen as an almost perfect target since it is well anchored to the B-cell membrane and does not undergo shedding or internalization. Furthermore, CD20 is expressed only in B-cells giving anti-CD20 MAbs a specificity rarely seen with other drugs, representing the goal of the so-called targeted therapy. Not without surprise, several anti-CD20 MAbs continue undergoing clinical development. Few examples include veltuzumab (IMMU-106), which is a humanized anti-CD20 MAb under development by Immunomedics Inc., and afutuzumab (GA-101), a third-generation anti-CD20 MAb with increased ADCC activity, currently under development by Genentech and Biogen Idec Inc. These drugs are undergoing phase I/II trials and have shown to be both safe and efficacious in patients with lymphoproliferative disorders. ${ }^{34,35}$

Ofatumumab, a second-generation anti-CD20 MAb, has emerged as a therapeutic option in patients with CLL. Several clinical trials have shown efficacy in frontline and relapsed settings, even after rituximab-containing regimens. Most importantly, responses and clinical improvements have been seen in patients with disease resistant to fludarabine and alemtuzumab and patients with bulky lymphadenopathy. It is worth emphasizing that these effects have been seen with ofatumumab administered as a single agent. The recent approval of ofatumumab for the treatment of CLL patients who have failed fludarabine- and alemtuzumab-containing regimens emphasizes its use as a third- or fourth-line therapy.

However, ofatumumab has a great potential for further development. An ongoing trial is evaluating the addition of ofatumumab to chlorambucil in untreated CLL patients and a second trial is studying its combination with fludarabine and cyclophosphamide in previously treated CLL patients. It is likely that, if these trials show positive results, ofatumumab will obtain approval at earlier stages of therapy. If the 
pre-clinical characteristics of ofatumumab such as stronger CDC and reduced off-rates will translate into a superior response or survival rates than rituximab is unclear at the moment and will need to be addressed in carefully designed comparative trials. Practitioners have used rituximab for several years with a high level of comfort; hence, it will be difficult to replace it unless the new therapy is associated with a better efficacy rate and safer toxicity profile. So far, infusion-related reactions seemed to occur less frequently with ofatumumab, and given its fully human structure it should be less immunogenic, allowing for faster intravenous infusions. Interesting concepts such as re-treatment and maintenance therapy with ofatumumab are under investigation; appropriately large study populations and long follow-up time are necessary in order to guarantee reliable conclusions.

Several agents targeting novel pathways are undergoing clinical development in patients with CLL. These will make available to us, the practitioners, potential combinations that hopefully will translate, if not into a cure, into better, more durable responses and longer survival times. Personalization of therapy seems inevitable for patients with CLL, most of which will experience a protracted clinical course and survival measured in several years. Therefore, the careful tailoring of our therapies should allow us to obtain such responses without affecting the quality of life of our patients.

In summary, ofatumumab, thus far, has demonstrated promising potential as a therapeutic option in relapsed CLL, even in patients who have already failed the most effective available therapies. Careful design, execution and analysis of prospective studies will be necessary to further refine and define the role of ofatumumab in standard clinical care in patients with CLL.

\section{Disclosures}

The authors have no conflicts of interest to disclose.

\section{References}

1. Jemal A, Siegel R, Ward E, et al. Cancer statistics. CA Cancer J Clin. 2009;59(4):225-249.

2. Hallek M, Cheson BD, Catovsky D, et al. Guidelines for the diagnosis and treatment of chronic lymphocytic leukemia: a report from the International Workshop on Chronic Lymphocytic Leukemia updating the National Cancer Institute-Working Group 1996 guidelines. Blood. 2008;111(12):5446-5456.

3. Van Bockstaele F, Verhasselt B, Philippe J. Prognostic markers in chronic lymphocytic leukemia: a comprehensive review. Blood Rev. 2009;23(1):25-47.

4. Rawstron AC, Bennett FL, O'Connor SJ, et al. Monoclonal B-cell lymphocytosis and chronic lymphocytic leukemia. $N$ Engl J Med. 2008;359(6):575-583.
5. Grever MR, Lucas DM, Dewald GW, et al. Comprehensive assessment of genetic and molecular features predicting outcome in patients with chronic lymphocytic leukemia: results from the US Intergroup Phase III Trial E2997. J Clin Oncol. 2007;25(7):799-804.

6. Eichhorst BF, Busch R, Stilgenbauer S, et al. First-line therapy with fludarabine compared with chlorambucil does not result in a major benefit for elderly patients with advanced chronic lymphocytic leukemia. Blood. 2009;114(16):3382-3391.

7. Rai KR, Peterson BL, Appelbaum FR, et al. Fludarabine compared with chlorambucil as primary therapy for chronic lymphocytic leukemia. N Engl J Med. 2000;343(24):1750-1757.

8. Knauf WU, Lissichkov T, Aldaoud A, et al. Phase III randomized study of bendamustine compared with chlorambucil in previously untreated patients with chronic lymphocytic leukemia. J Clin Oncol. 2009;27(26):4378-4384.

9. Stilgenbauer S, Dohner H. Campath-1H-induced complete remission of chronic lymphocytic leukemia despite p53 gene mutation and resistance to chemotherapy. N Engl J Med. 2002;347(6):452-453.

10. Lozanski G, Heerema NA, Flinn IW, et al. Alemtuzumab is an effective therapy for chronic lymphocytic leukemia with p53 mutations and deletions. Blood. 2004;103(9):3278-3281.

11. Moreton P, Kennedy B, Lucas G, et al. Eradication of minimal residual disease in B-cell chronic lymphocytic leukemia after alemtuzumab therapy is associated with prolonged survival. J Clin Oncol. 2005;23(13):2971-2979.

12. Keating MJ, Flinn I, Jain V, et al. Therapeutic role of alemtuzumab (Campath-1H) in patients who have failed fludarabine: results of a large international study. Blood. 2002;99(10):3554-3561.

13. Hallek M, Fingerle-Rowson G, Fink AM, et al. Immunochemotherapy with fludarabine $(\mathrm{F})$, cyclophosphamide (C), and rituximab (R) (FCR) versus fludarabine and cyclophosphamide (FC) improves response rates and progression-free survival (PFS) of previously untreated patients (pts) with advanced chronic lymphocytic leukemia (CLL). Blood (ASH Annual Meeting Abstracts). 2008;112(11): Abstract 325.

14. Robak T, Moiseev SI, Dmoszynska A, et al. Rituximab, fludarabine, and cyclophosphamide (R-FC) prolongs progression free survival in relapsed or refractory chronic lymphocytic leukemia (CLL) compared with FC Alone: final results from the international randomized phase III REACH Trial. Blood (ASH Annual Meeting Abstracts) 2008; 112(11):lba-1.

15. Byrd JC, Shinn C, Waselenko JK, et al. Flavopiridol induces apoptosis in chronic lymphocytic leukemia cells via activation of caspase-3 without evidence of bcl-2 modulation or dependence on functional p53. Blood. 1998;92(10):3804-3816.

16. Lin TS, Ruppert AS, Johnson AJ, et al. Phase II study of flavopiridol in relapsed chronic lymphocytic leukemia demonstrating high response rates in genetically high-risk disease. J Clin Oncol. 2009;27(35):6012-6018.

17. Wu L, Adams M, Carter T, et al. lenalidomide enhances natural killer cell and monocyte-mediated antibody-dependent cellular cytotoxicity of rituximab-treated CD20+ tumor cells. Clin Cancer Res. 2008;14(14):4650-4657.

18. Mitsiades CS, Mitsiades N. CC-5013 (Celgene). Curr Opin Investig Drugs. 2004;5(6):635-647.

19. Chanan-Khan A, Miller KC, Musial L, et al. Clinical efficacy of lenalidomide in patients with relapsed or refractory chronic lymphocytic leukemia: results of a phase II study. J Clin Oncol. 2006;24(34): 5343-5349.

20. Tam CS, O'Brien S, Lerner S, et al. The natural history of fludarabinerefractory chronic lymphocytic leukemia patients who fail alemtuzumab or have bulky lymphadenopathy. Leuk Lymphoma. 2007;48(10): 1931-1939.

21. Castillo J, Winer E, Quesenberry P. Newer monoclonal antibodies for hematological malignancies. Exp Hematol. 2008;36(7):755-768.

22. Castillo J, Milani C, Mendez-Allwood D. Ofatumumab, a secondgeneration anti-CD20 monoclonal antibody, for the treatment of lymphoproliferative and autoimmune disorders. Expert Opin Investig Drugs. 2009;18(4):491-500. 
23. Teeling JL, French RR, Cragg MS, et al. Characterization of new human CD20 monoclonal antibodies with potent cytolytic activity against non-Hodgkin lymphomas. Blood. 2004;104(6):1793-800.

24. Teeling JL, Mackus WJ, Wiegman LJ, et al. The biological activity of human CD20 monoclonal antibodies is linked to unique epitopes on CD20. J Immunol. 2006;177(1):362-371.

25. Beum PV, Lindorfer MA, Beurskens F, et al. Complement activation on B lymphocytes opsonized with rituximab or ofatumumab produces substantial changes in membrane structure preceding cell lysis. J Immunol. 2008;181(1):822-832.

26. Bleeker WK, Munk ME, Mackus WJ, et al. Estimation of dose requirements for sustained in vivo activity of a therapeutic human anti-CD20 antibody. Br J Haematol. 2008;140(3):303-312.

27. Coiffier B, Lepretre S, Pedersen LM, et al. Safety and efficacy of ofatumumab, a fully human monoclonal anti-CD20 antibody, in patients with relapsed or refractory B-cell chronic lymphocytic leukemia: a phase 1-2 study. Blood. 2008;111(3):1094-1100.

28. Osterborg A, Kipps TJ, Mayer J, et al. Ofatumumab (HuMax-CD20), a novel CD20 monoclonal antibody, is an active treatment for patients with CLL refractory to both fludarabine and alemtuzumab or bulky fludarabine-refractory disease: results from the planned interim analysis of an international pivotal trial. Blood (ASH Annual Meeting Abstracts). 2008;112(11): Abstract 328 .

29. Wierda WG, Kipps T, Mayer J, et al. Activity of ofatumumab, a novel $\mathrm{CD} 20 \mathrm{mAb}$, and prior rituximab exposure in patients with fludarabineand alemtuzumab-refractory or bulky fludarabine-refractory chronic lymphocytic leukemia (CLL). ASCO Meeting Abstracts. 2009;27(15S): Abstract 7044.

30. Kipps TJ, Osterborg A, Mayer J, et al. Clinical improvement with a novel CD20 mAb, ofatumumab, in fludarabine-refractory chronic lymphocytic leukemia (CLL) also refractory to alemtuzumab or with bulky lymphadenopathy. ASCO Meeting Abstracts. 2009;27(15S): Abstract 7043.

31. ClinicalTrials.gov. A Service of the US National Institutes of Health. Available at www.clinicaltrials.gov. Accessed on November 17, 2009.

32. Wierda WG, Kipps TJ, Dürig J, et al. Ofatumumab combined with fludarabine and cyclophosphamide (O-FC) shows high activity in patients with previously untreated chronic lymphocytic leukemia (CLL): results from a randomized, multicenter, international, two-dose, parallel group, phase ii trial. Blood (ASH Annual Meeting Abstracts). 2009;114(22): Abstract 207.

33. FDA Approves New Treatment for Chronic Lymphocytic Leukemia. Released October 26, 2009. Available at http:/www.fda. gov/NewsEvents/Newsroom/PressAnnouncements/ucm187966.htm. Accessed Nov 17, 2009.

34. Milani C, Castillo J. Veltuzumab, an anti-CD20 mAb for the treatment of non-Hodgkin's lymphoma, chronic lymphocytic leukemia and immune thrombocytopenic purpura. Curr Opin Mol Ther. 2009;11(2):200-207.

35. Robak T. GA-101, a third-generation, humanized and glyco-engineered anti-CD20 $\mathrm{mAb}$ for the treatment of B-cell lymphoid malignancies. Curr Opin Investig Drugs. 2009;10(6):588-596.
36. Hillmen P, Skotnicki AB, Robak T, et al. Alemtuzumab compared with chlorambucil as first-line therapy for chronic lymphocytic leukemia. J Clin Oncol. 2007;25(35):5616-5623.

37. Hallek M, Fingerle-Rowson G, Fink AM, et al; First-line treatment with fludarabine (F), cyclophosphamide (C), and rituximab (R) (FCR) improves overall survival (OS) in previously untreated patients (pts) with advanced chronic lymphocytic leukemia (CLL): results of a randomized phase III trial on behalf of an international group of investigators and the German CLL Study Group. Blood (ASH Annual Meeting Abstracts). 2009;114(22): Abstract 535.

38. Robak T, Blonski JZ, Gora-Tybor J, et al. Cladribine alone and in combination with cyclophosphamide or cyclophosphamide plus mitoxantrone in the treatment of progressive chronic lymphocytic leukemia: report of a prospective, multicenter, randomized trial of the Polish Adult Leukemia Group (PALG CLL2). Blood. 2006;108(2): 473-479.

39. Kay NE, Geyer SM, Call TG, et al. Combination chemoimmunotherapy with pentostatin, cyclophosphamide, and rituximab shows significant clinical activity with low accompanying toxicity in previously untreated B chronic lymphocytic leukemia. Blood. 2007;109(2):405-411.

40. Parikh SA, Keating M, O'Brien S, et al. Frontline combined chemoimmunotherapy with fludarabine, cyclophosphamide, alemtuzumab and rituximab (CFAR) in high-risk chronic lymphocytic leukemia. Blood (ASH Annual Meeting Abstracts). 2009;114(22): Abstract 208.

41. Fischer K, Cramer P, Stilgenbauer S, et al. Bendamustine combined with rituximab (BR) in first-line therapy of advanced CLL: a multicenter phase II trial of the German CLL Study Group (GCLLSG). Blood (ASH Annual Meeting Abstracts). 2009;114(22): Abstract 205.

42. O'Brien S, Moore JO, Boyd TE, et al. Randomized phase III trial of fludarabine plus cyclophosphamide with or without oblimersen sodium (Bcl-2 antisense) in patients with relapsed or refractory chronic lymphocytic leukemia. J Clin Oncol. 2007;25(9):1114-1120.

43. O'Brien S, Moore JO, Boyd TE, et al. 5-year survival in patients with relapsed or refractory chronic lymphocytic leukemia in a randomized, phase III trial of fludarabine plus cyclophosphamide with or without oblimersen. J Clin Oncol. 2009;27(31):5208-5212.

44. Fischer K, Stilgenbauer S, Schweighofer CD, et al. Bendamustine in combination with rituximab (BR) for patients with relapsed chronic lymphocytic leukemia (CLL): a multicentre phase ii trial of the German CLL Study Group (GCLLSG) Blood. (ASH Annual Meeting Abstracts). 2008; 112(11): Abstract 330.

45. Byrd JC, Kipps TJ, Flinn IW, et al. Phase 1/2 study of lumiliximab combined with fludarabine, cyclophosphamide, and rituximab in patients with relapsed or refractory chronic lymphocytic leukemia. Blood. 2010; 115(3):489-495.

46. Lamanna N, Kalaycio M, Maslak P, et al. Pentostatin, cyclophosphamide, and rituximab is an active, well-tolerated regimen for patients with previously treated chronic lymphocytic leukemia. J Clin Oncol. 2006;24(10):1575-1581.
Journal of Blood Medicine

\section{Publish your work in this journal}

The Journal of Blood Medicine is an international, peer-reviewed, open access, online journal publishing laboratory, experimental and clinical aspects of all topics pertaining to blood based medicine including but not limited to: Transfusion Medicine; Blood collection, Donor issues, Transmittable diseases, and Blood banking logistics; Immunohematology; Artificial and alternative blood

\section{Dovepress}

based therapeutics; Hematology; Biotechnology/nanotechnology of blood related medicine; Legal aspects of blood medicine; Historical perspectives. The manuscript management system is completely online and includes a very quick and fair peer-review system. Visit http://www.dovepress.com/ testimonials.php to read real quotes from published authors. 